\title{
Unusual Cerebral Infarct Patterns Every Radiologist Should Know
}

Stephanie Wu, MD MBA

Mougnyan Cox, MD

Seyed Ali Nabavizadeh, MD

Penn Medicine 


\section{Purpose}

- Review ten unusual cases of brain infarcts associated with infarcts of the anterior cerebral circulation, posterior cerebral circulation, and brainstem stroke syndromes.

- Vital for radiologists to be able to recognize more uncommon patterns of cerebral infarctions.

- Cerebral infarction is a leading cause of morbidity and mortality worldwide. Establishing accurate and timely diagnoses in patients with acute brain infarcts is crucial to patient care and ultimately patient prognosis. 


\section{Outline}

- Infarcts in the anterior cerebral circulation

- Anterior choroidal artery

- Recurrent artery of Heubner

- Pericallosal artery

- Lateral lenticulostriate artery

- Precentral gyrus infarct

- Infarcts in the posterior cerebral circulation

- Artery of Percheron

- Choroid plexus

- Brainstem stroke syndromes

- Medial longitudinal fasciculus infarct with internuclear ophthalmoplegia

- Facial colliculus syndrome

- Lateral medullary syndrome (Wallenberg) 
Infarcts in the Anterior Cerebral Circulation 


\section{Anterior Choroidal Artery Infarct}

\section{- Anatomy of anterior choroidal artery}

- Anterior choroidal artery originates from posterior wall of ICA between origin of posterior communicating artery and internal carotid terminus

- Vascular territory

- Deep brain structures

- Posterior limb of internal capsule, lateral thalamus

- Optic tract, lateral cerebral peduncle

- Globus pallidus internus, tail of caudate nucleus

- Mesial temporal structures: Hippocampus head, amygdala

- Choroid plexus of anterior part of temporal horns of lateral ventricles

\section{- Clinical Presentation}

- Complete triad: Hemiplegia, hemianesthesia, and contralateral hemianopia

- Rare due to anastomosis with MCA, PCA, and posterior communicating artery

- Typically associated with carotid stenosis and cardioembolic sources

- Incomplete forms of anterior choroidal artery infarcts are more common (lacunar syndromes)

- Typically lacunar strokes secondary to hypertension, diabetes, and hyperlipidemia

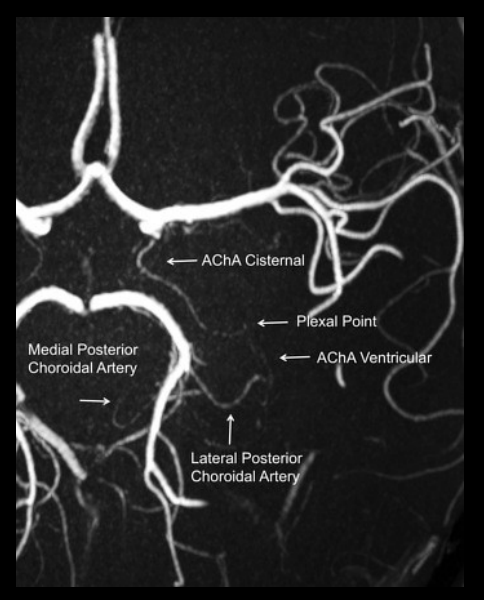




\section{Anterior Choroidal Artery Infarct}

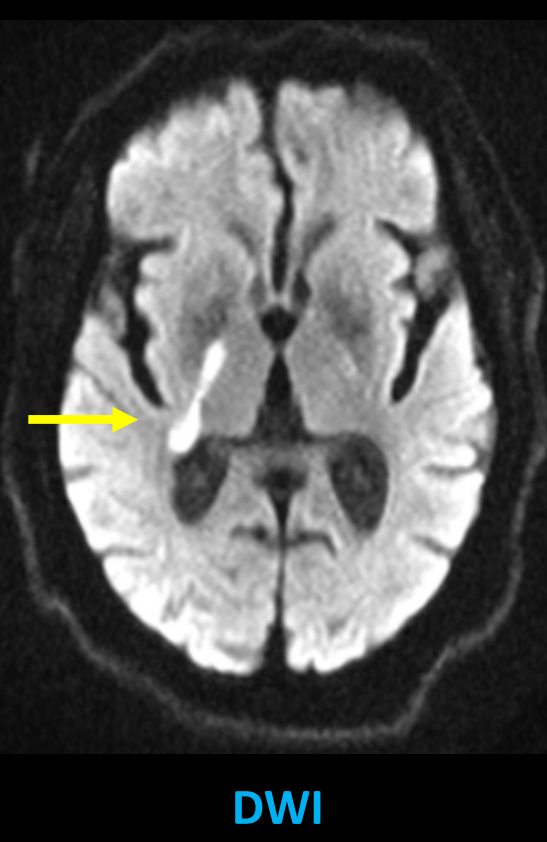

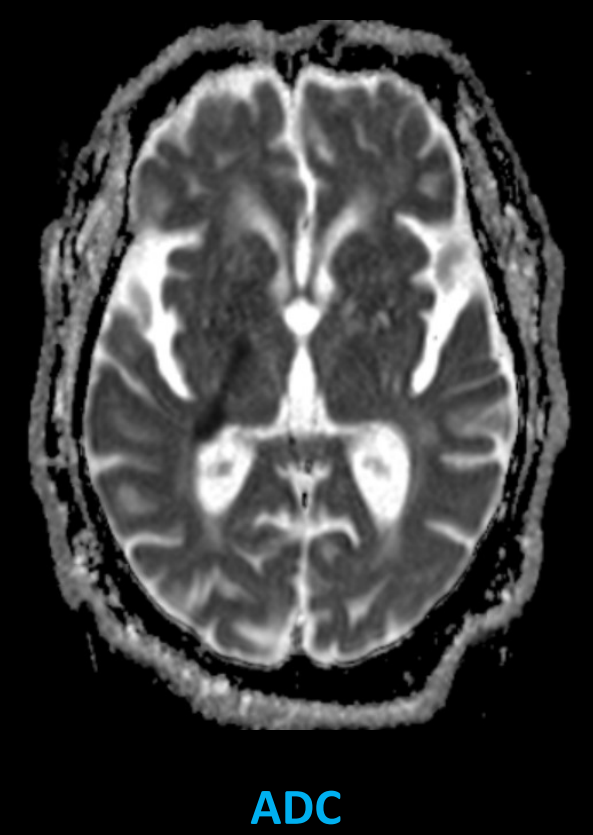

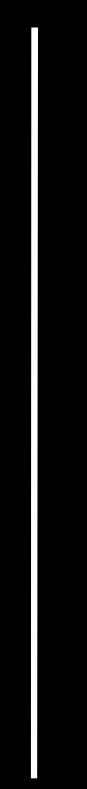

80 yo $\mathrm{M}$ presenting with L-sided weakness, $\mathrm{R}$ gaze preference, and $\mathrm{R}$ visual field deficit.

Acute right anterior choroidal artery infarct involving the posterior limb of right internal capsule, extending posterolaterally to the ventricular margin. There is also involvement of the right medial temporal lobe. 


\section{Anterior Choroidal Artery Infarct}

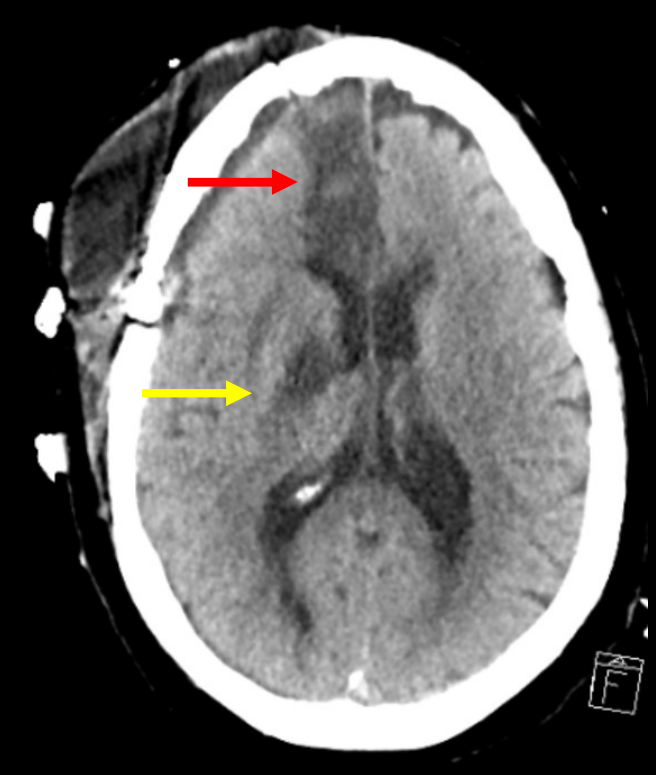

CT

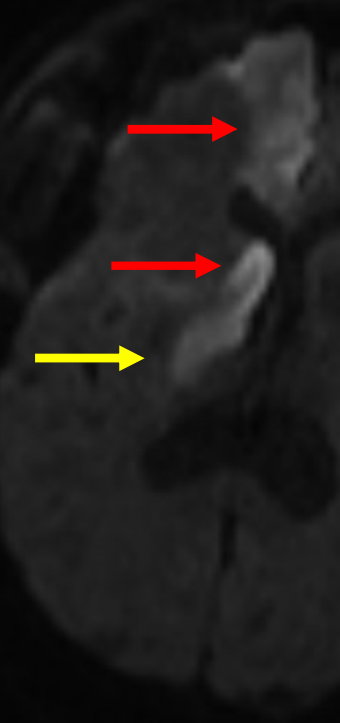

DWI

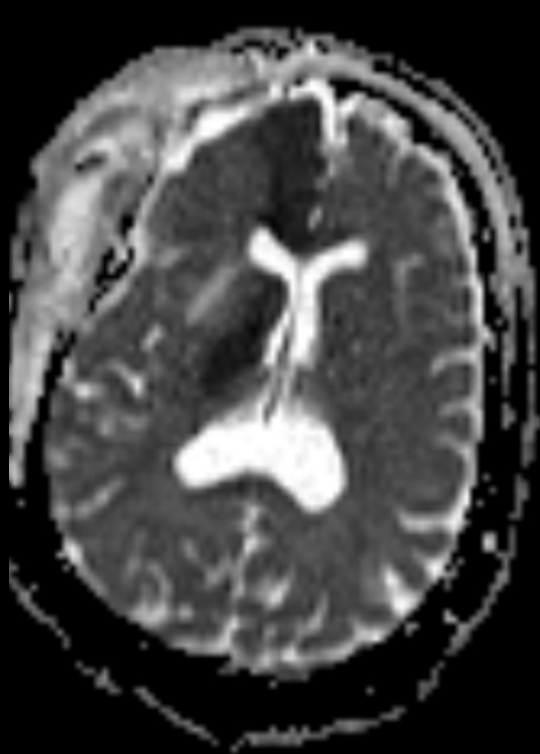

ADC

62 yo F with bilateral giant ICA aneurysms, s/p surgical clipping of R ICA terminus and R anterior choroidal artery aneurysms.

Acute right anterior choroidal artery infarct involving the posterior limb of right internal capsule. Additional right ACA territory infarct involving the medial right frontal lobe and right caudate head. Post-surgical changes from right craniotomy with pseudomeningocele and right frontal subdural collection. 


\section{Anterior Choroidal Artery Infarct}

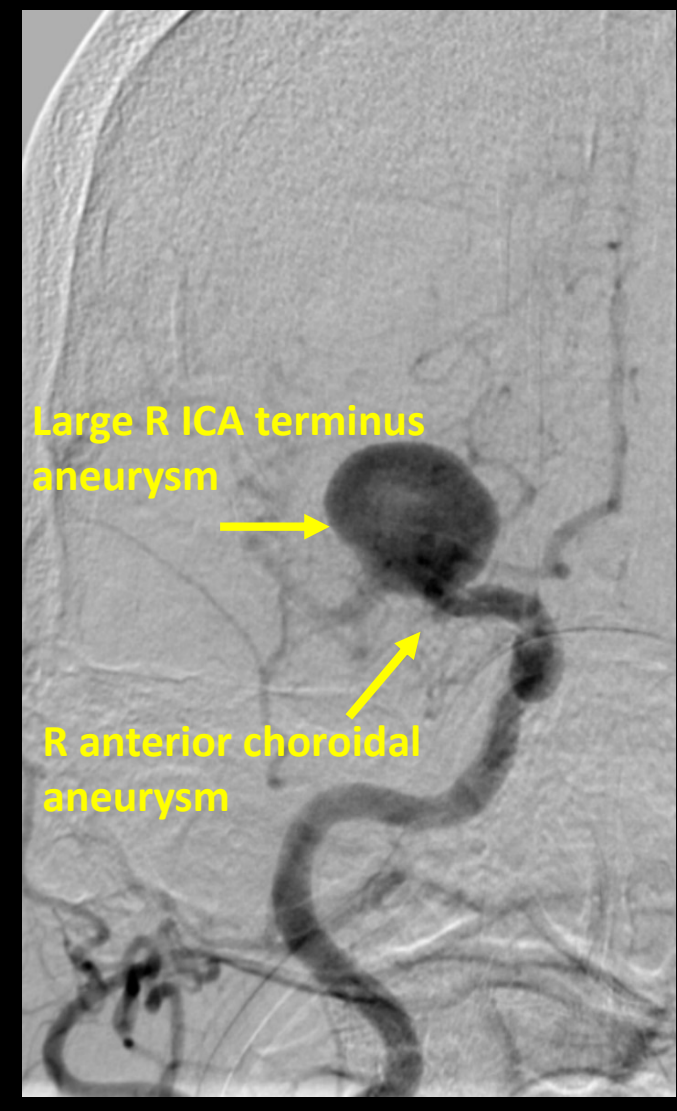

Pre-Aneurysm Clipping Frontal Right CCA Injection

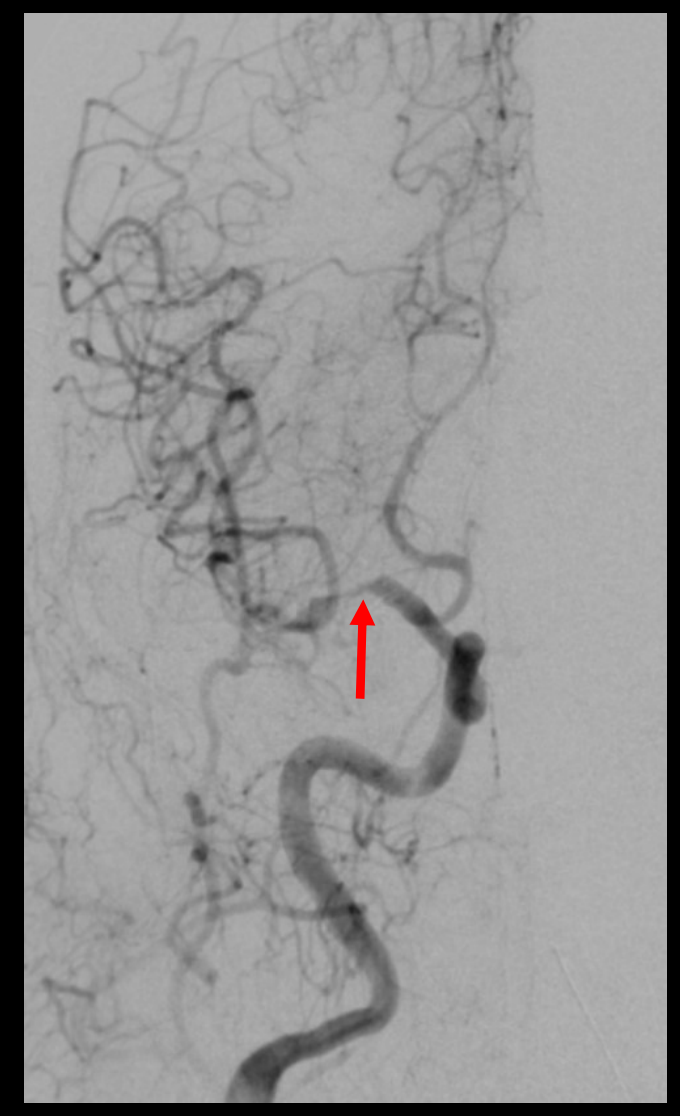

Post-Aneurysm Clipping

No contrast filling of surgically clipped right ICA terminus or right anterior choroidal aneurysms.

Severe stenosis of right M1 segment. Narrowing of right A1 segment and distal right ACA branches. 


\section{Recurrent Artery of Heubner Infarct}

- Anatomy

- Recurrent artery of Heubner is a prominent medial lenticulostriate branch typically arising from distal A1 or proximal A2 segment of anterior cerebral artery

- Supplies head of caudate, anterior lentiform nucleus, and anterior limb of internal capsule

- "Recurrent" since it goes back laterally along the A1 segment towards terminal ICA $\rightarrow$ risk of injury from ACOM aneurysm clipping

- Clinical Presentation

- Weakness of the contralateral face and arm

- Dysarthria due to involvement of corticolingual and corticostriatocerebellar pathways

- Hemichorea

- Aphasia in left-sided involvement, left visual neglect in right-sided involvement

\section{- Etiologies}

- Large artery disease (e.g. stenosis of carotid artery), small vessel disease, cardiac emboli

- Trauma

- Vasospasm after ruptured aneurysm, inadvertent clipping during clipping of ACOM aneurysm

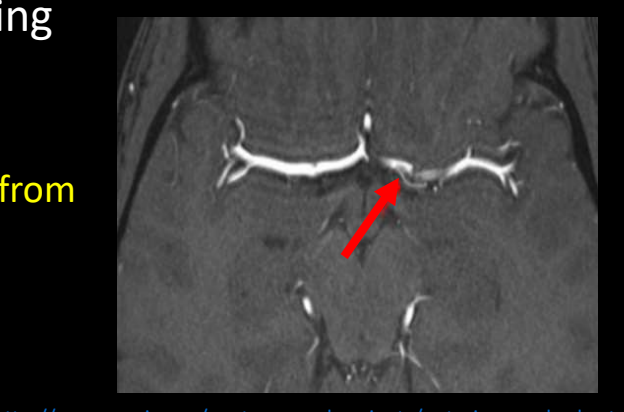




\section{Recurrent Artery of Heubner Infarct}

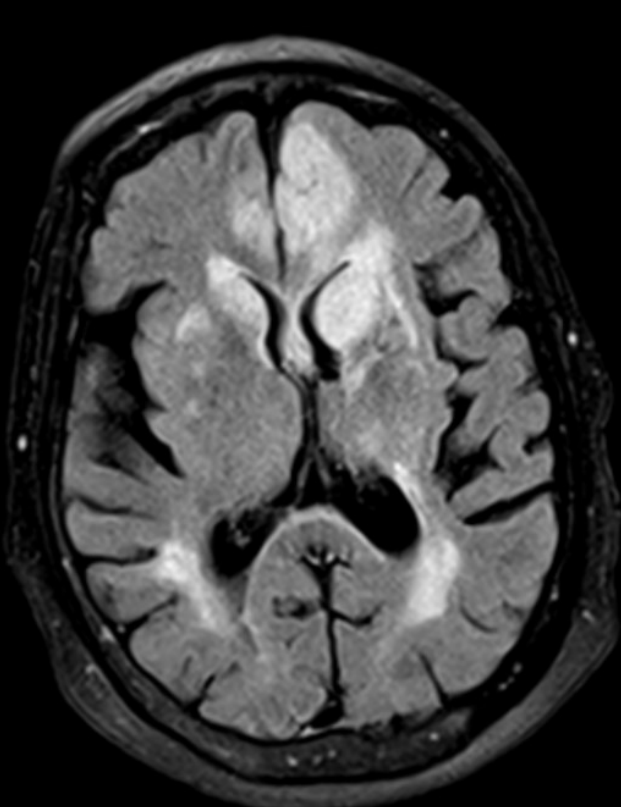

FLAIR

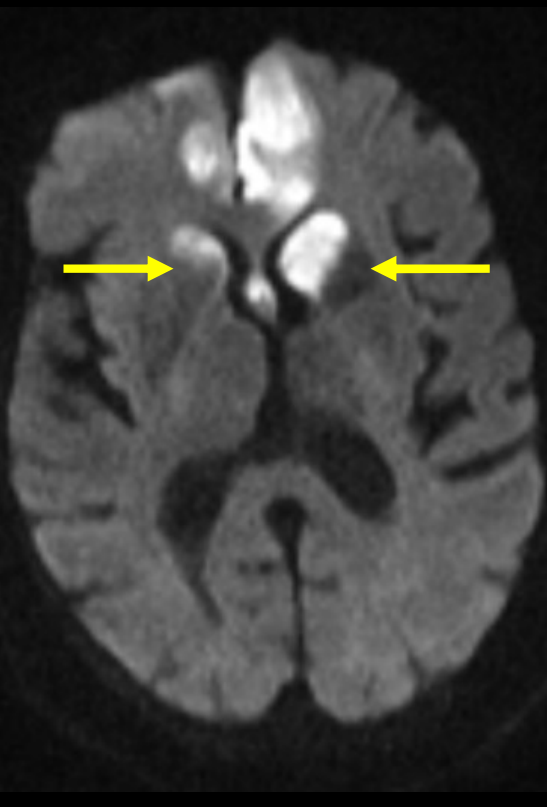

DWI

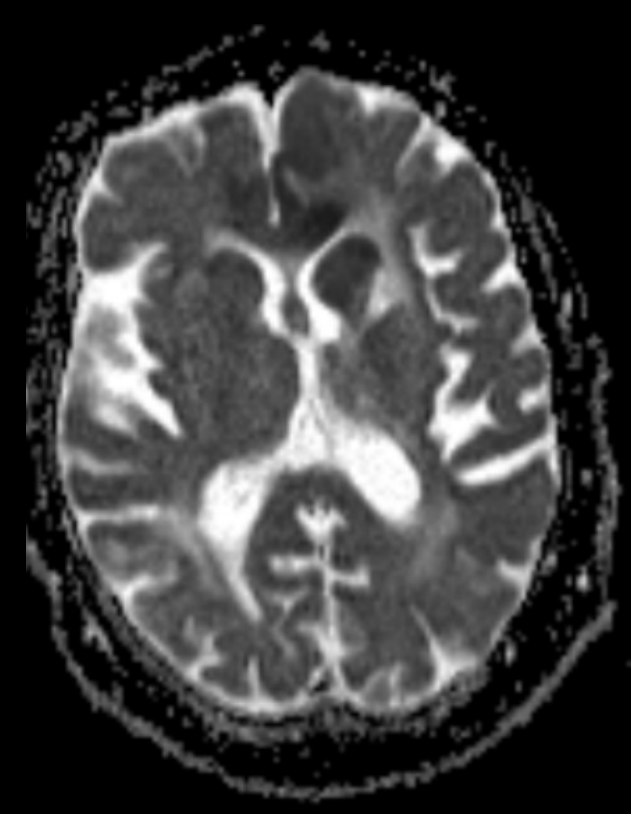

ADC

66 yo $\mathrm{F}$, presenting with aphasia, altered mental status, and difficulty following commands.

Acute bilateral ACA territory infarcts involving the medial frontal lobes and bilateral caudate heads (involvement of bilateral recurrent arteries of Heubner). 


\section{Pericallosal Arterial Infarct}

- Anatomy of pericallosal artery

- Distal portion of anterior cerebral artery extending posteriorly in the pericallosal sulcus

- Clinical Presentation

- Contralateral hemiparesis with lower limb predominance

- Involvement of supplementary motor cortex. Corticospinal tract typically intact

- Etiology

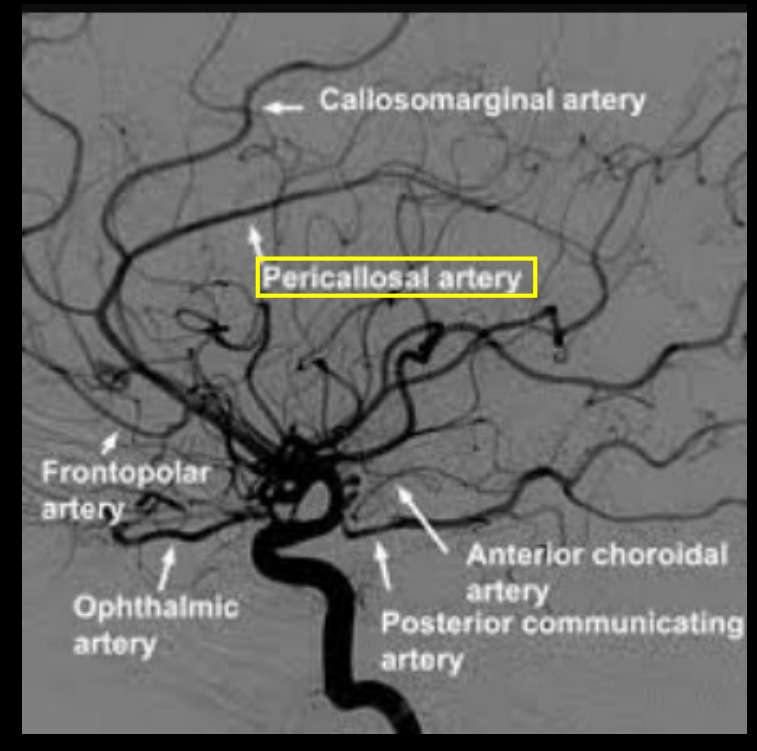

- Cardiac embolism, atherosclerotic disease 


\section{Pericallosal Arterial Infarct}

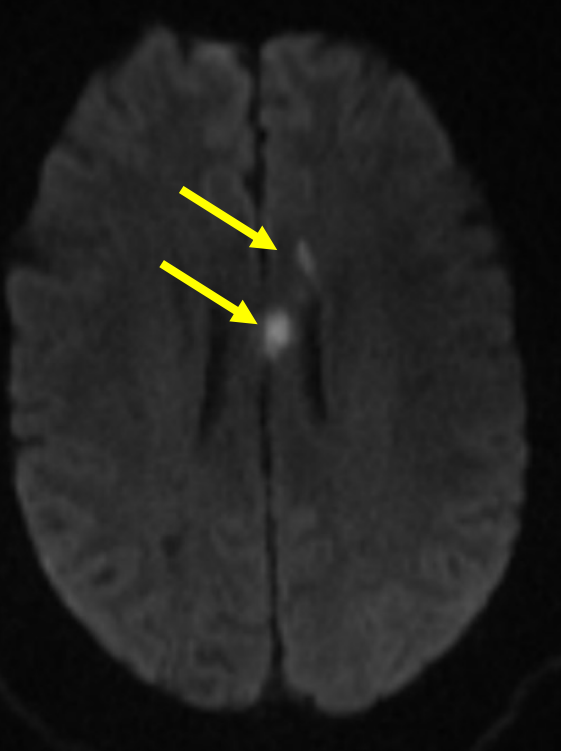

DWI

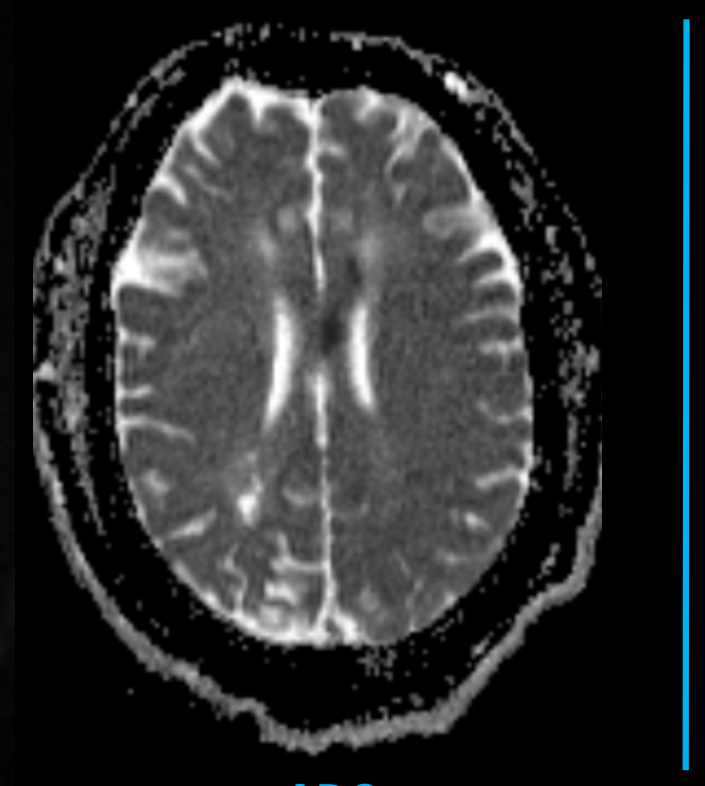

ADC

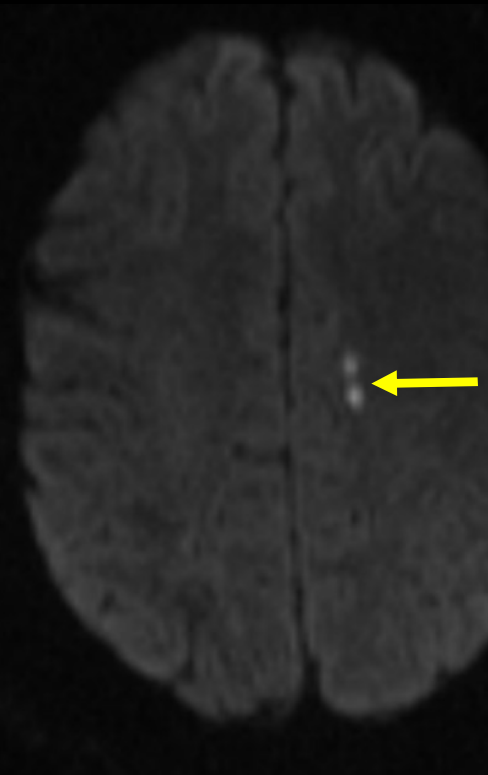

DWI

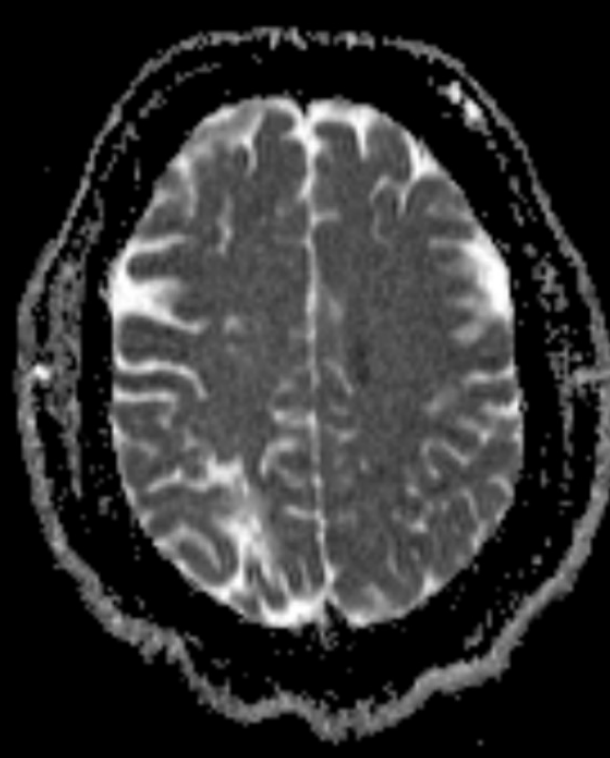

ADC

36 yo $\mathrm{M}$ with history of prior cerebral infarcts, presenting with vertigo, gait instability, and left leg dysmetria. Acute left corpus callosal and pericallosal infarcts. 


\section{Pericallosal Arterial Infarct}

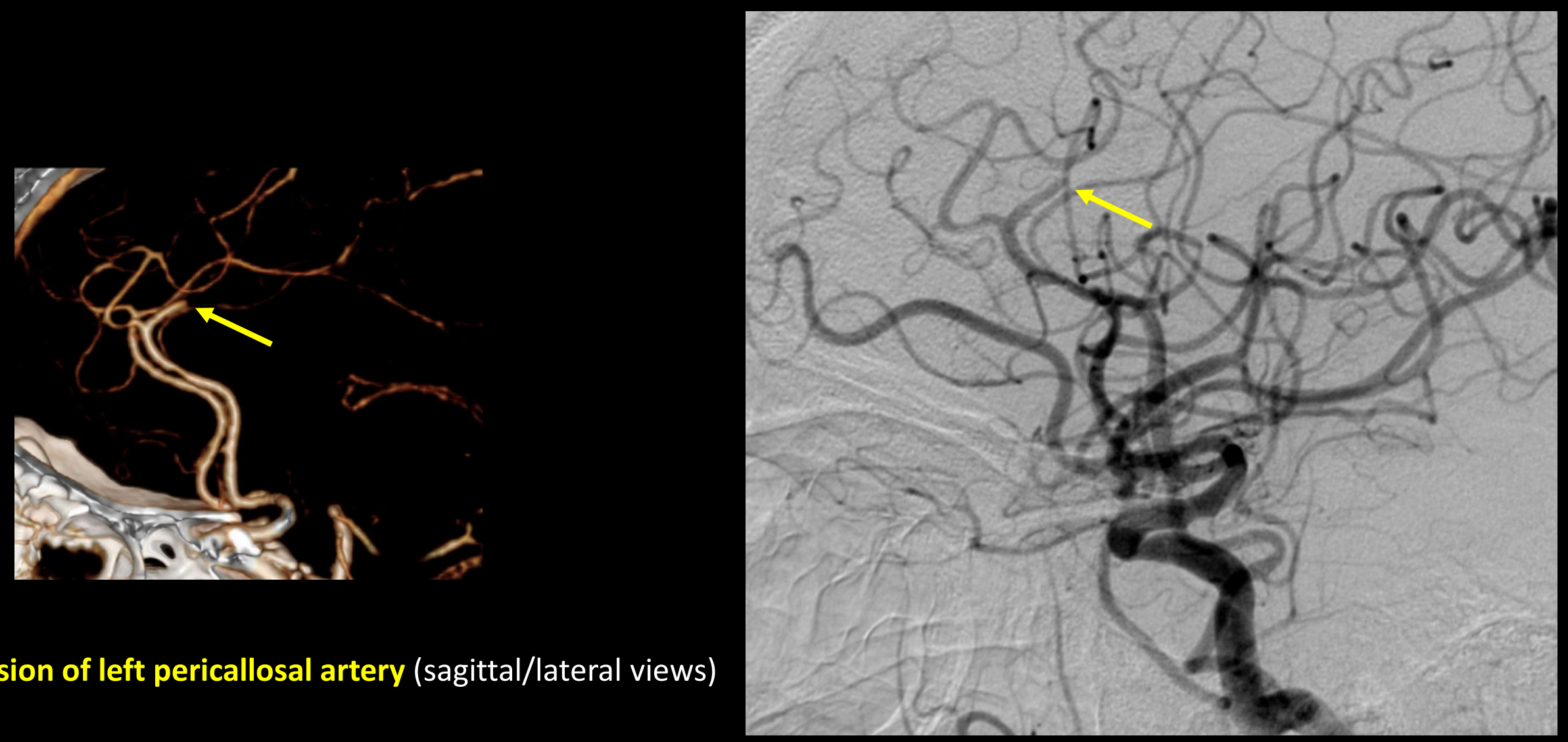




\section{Lateral Lenticulostriate Arterial Infarct}

- Anatomy

- Lateral lenticulostriate arteries typically branch off of M1 segment of middle cerebral artery (more rarely from M2)

- Supply lateral putamen, external capsule, and upper internal capsule

- Clinical Presentation

- Contralateral hemiplegia and sensory deficits

- Speech may be affected (medial temporal lobe)

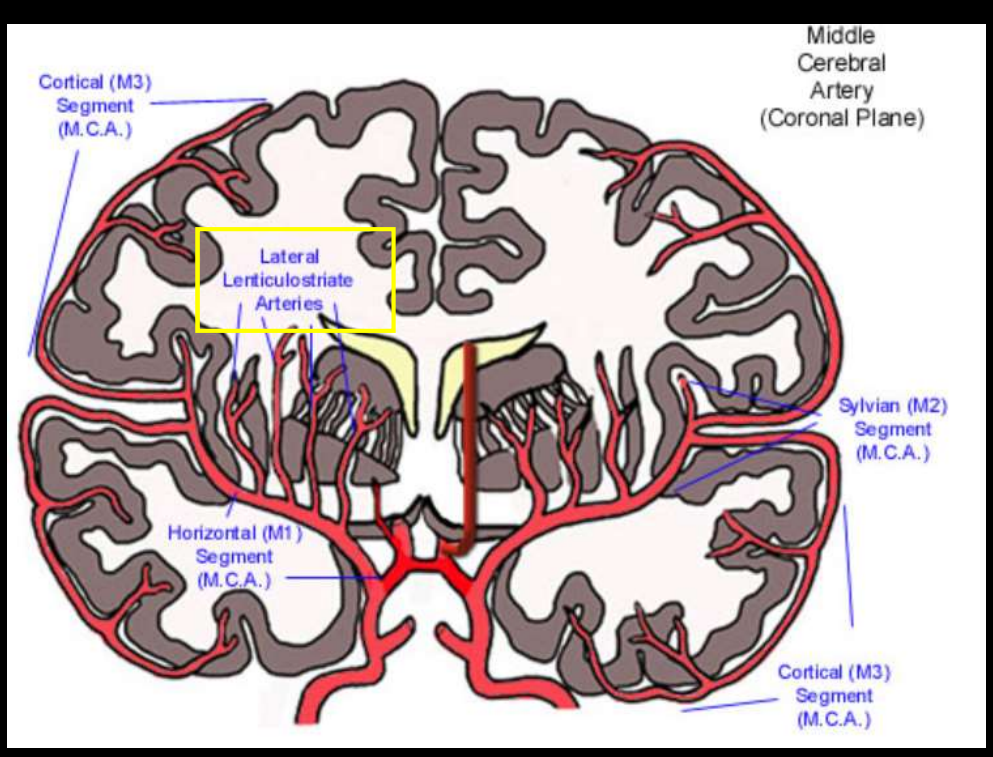




\section{Lateral Lenticulostriate Arterial Infarct}

73 yo F, presenting with left facial and left extremity weakness.

Infarct centered in the posterior right putamen extending to the right corona radiata and caudate body (lateral lenticulostriate artery territory).
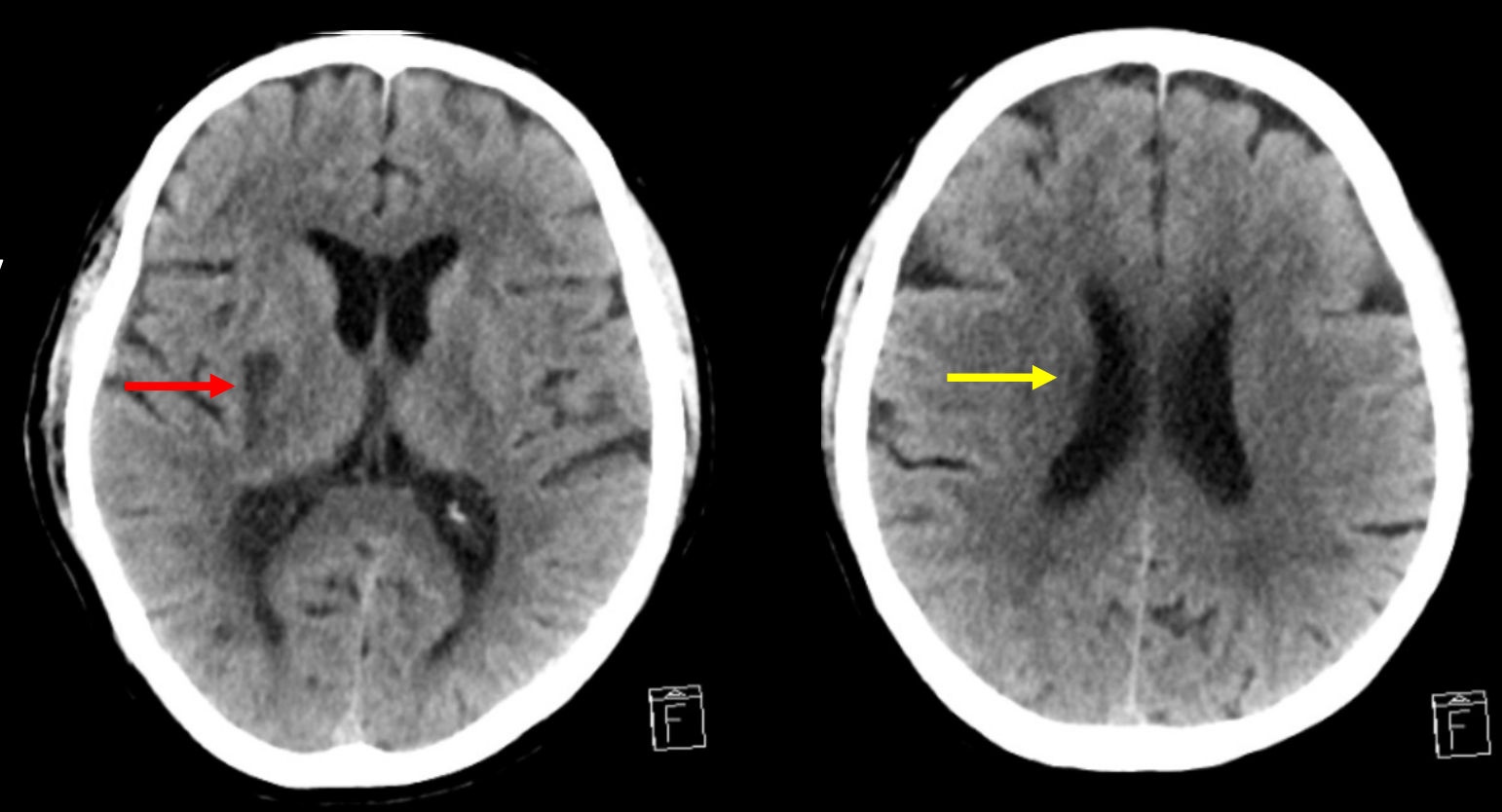


\section{Lateral Lenticulostriate Arterial Infarct}

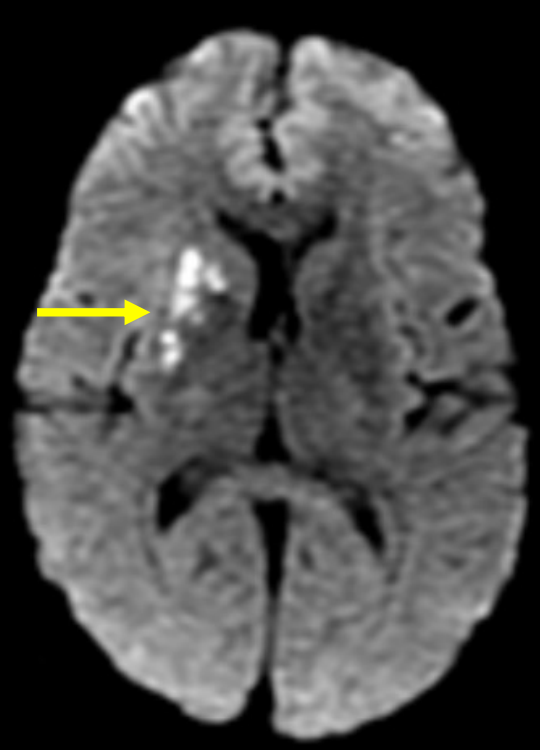

DWI

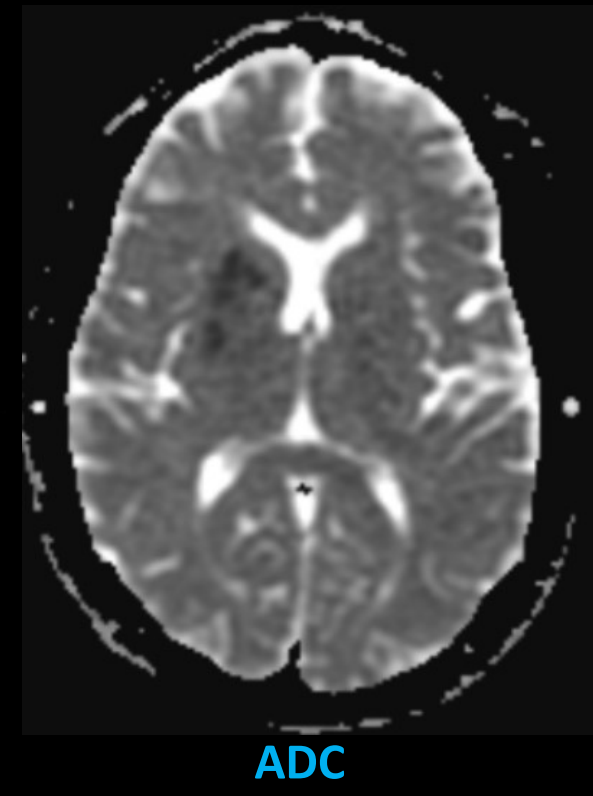

51 yo, presenting from outside institution.

Infarct in the right subinsular region extending into right putamen.

The next slide illustrates findings from this patient's MRA and follow-up conventional angiogram. 


\section{Lateral Lenticulostriate Arterial Infarct}

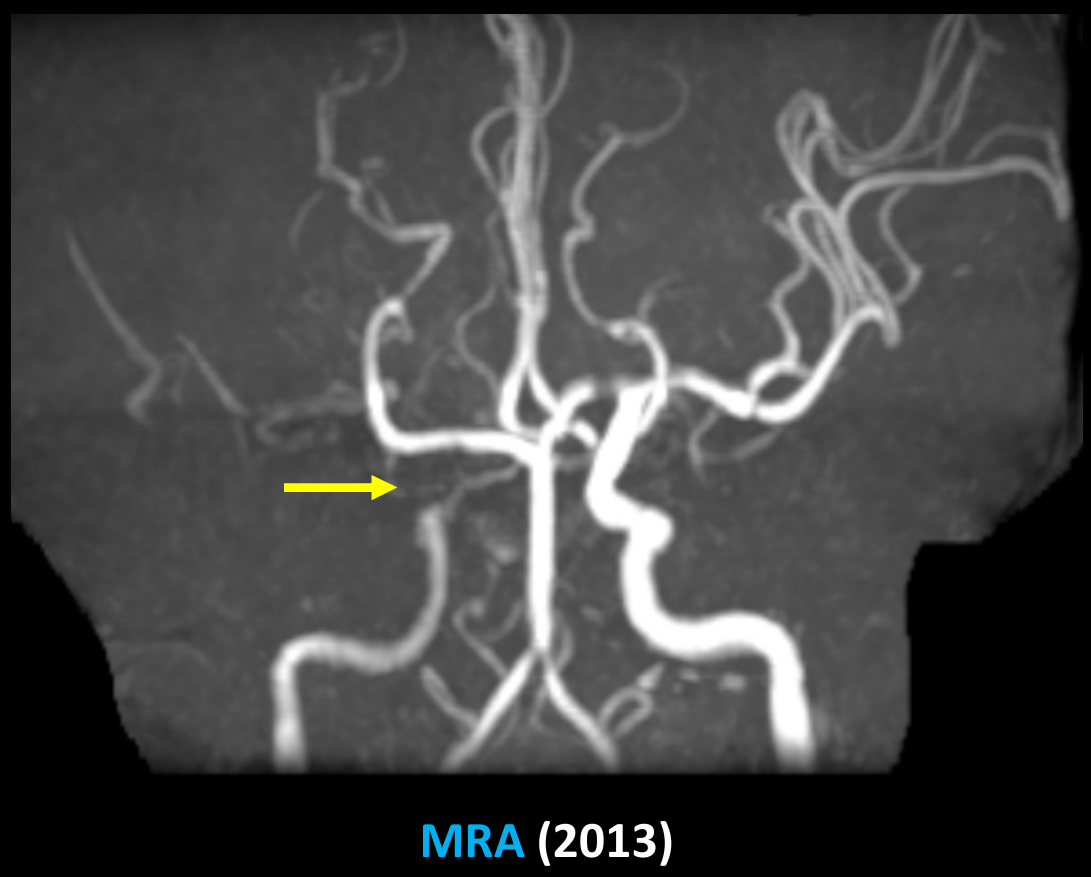

Diminutive right distal ICA with right carotid terminus occlusion

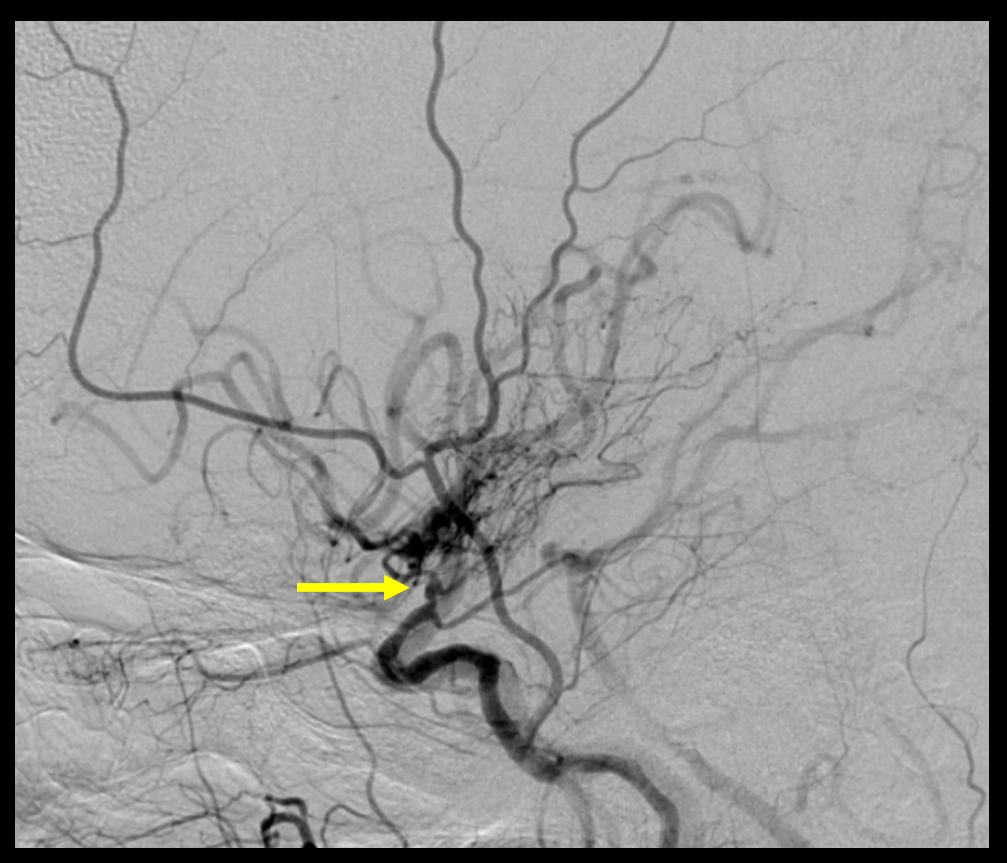

Lateral right CCA Injection (2018)

- Occlusion of the right ICA terminus.

- Reconstitution of right MCA territory via numerous tortuous collaterals from lateral lenticulostriate arteries and posterior pericallosal arteries. 


\section{Precentral Gyrus Infarct}

- Anatomy

- Precentral gyrus is anatomical location of primary motor cortex (responsible for contralateral voluntary motor movement via Betz cells)

- Predominant arterial blood supply from branches of middle cerebral artery

- Anterior cerebral artery supplies medial aspect of precentral gyrus (legs in cortical homunculus)

- Origin of corticospinal and corticobulbar tracts

\section{- Clinical Presentation}

- Paralysis of contralateral body (facial palsy, hemiparesis)

- Typically central facial palsy (unilateral facial paralysis with forehead sparing), although there have been reports of peripheral-type facial palsy with precentral gyrus infarct

- Damage to left precentral gyrus has been associated with speech apraxia

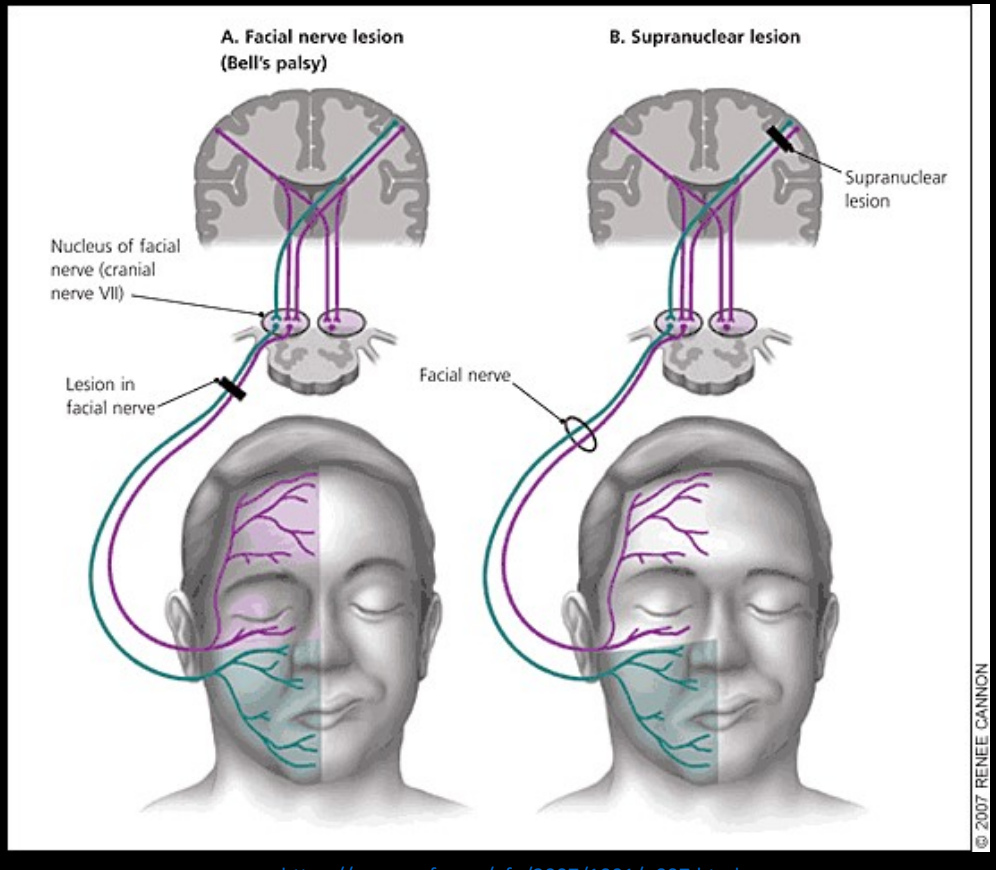




\section{Precentral Gyrus Infarct}

87 yo F, presenting with left facial droop (sparing of forehead).

Acute infarct centered in the right pre-central gyrus extending to the centrum semiovale.

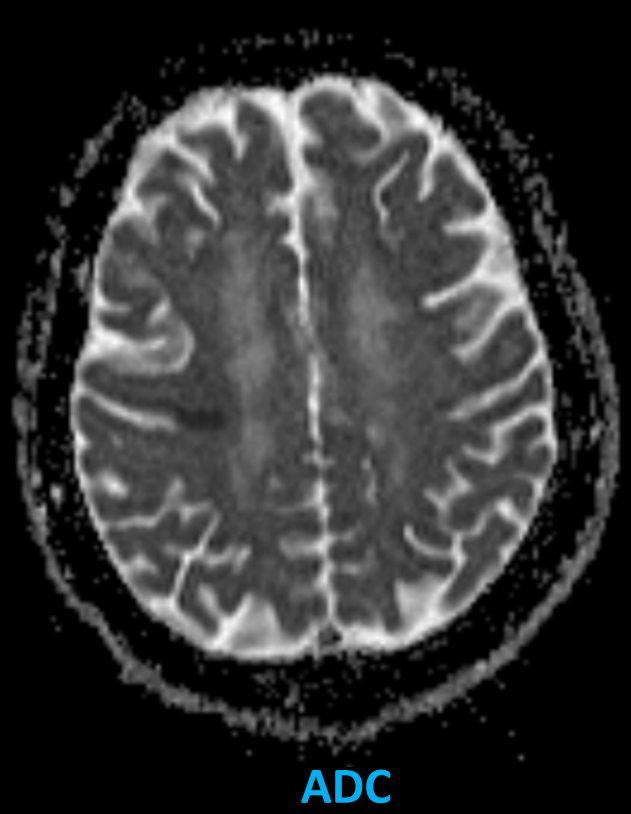


Infarcts in the Posterior Cerebral Circulation 


\section{Artery of Percheron Infarct}

- Anatomy

- Artery of Percheron: variant of posterior cerebral circulation

- Single thalamoperforating artery (branch of P1) supplying blood to paramedian thalami bilaterally and often the rostral midbrain

- Clinical Presentation

- (Thalamic involvement) AMS, vertical gaze palsy, and memory impairment

- (Rostral midbrain involvement) Additional oculomotor disturbances, hemiplegia, cerebellar ataxia, and movement disorders

- Imaging

- (Most common) Bilateral paramedian thalamic and midbrain infarcts

- Midbrain "V sign": V-shaped hyperintensity along pial surface of midbrain at interpeduncular fossa

- Can also see infarct of anterior thalami
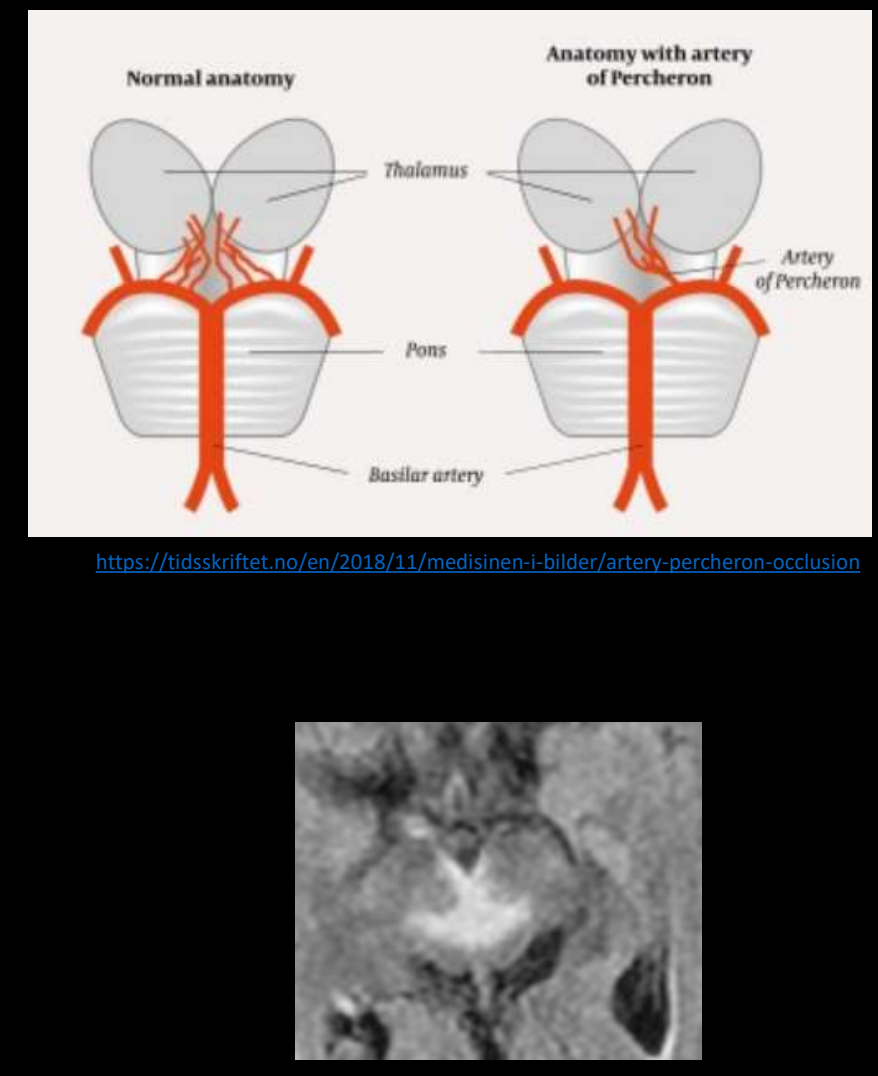

Midbrain "V sign" 


\section{Artery of Percheron Infarct}

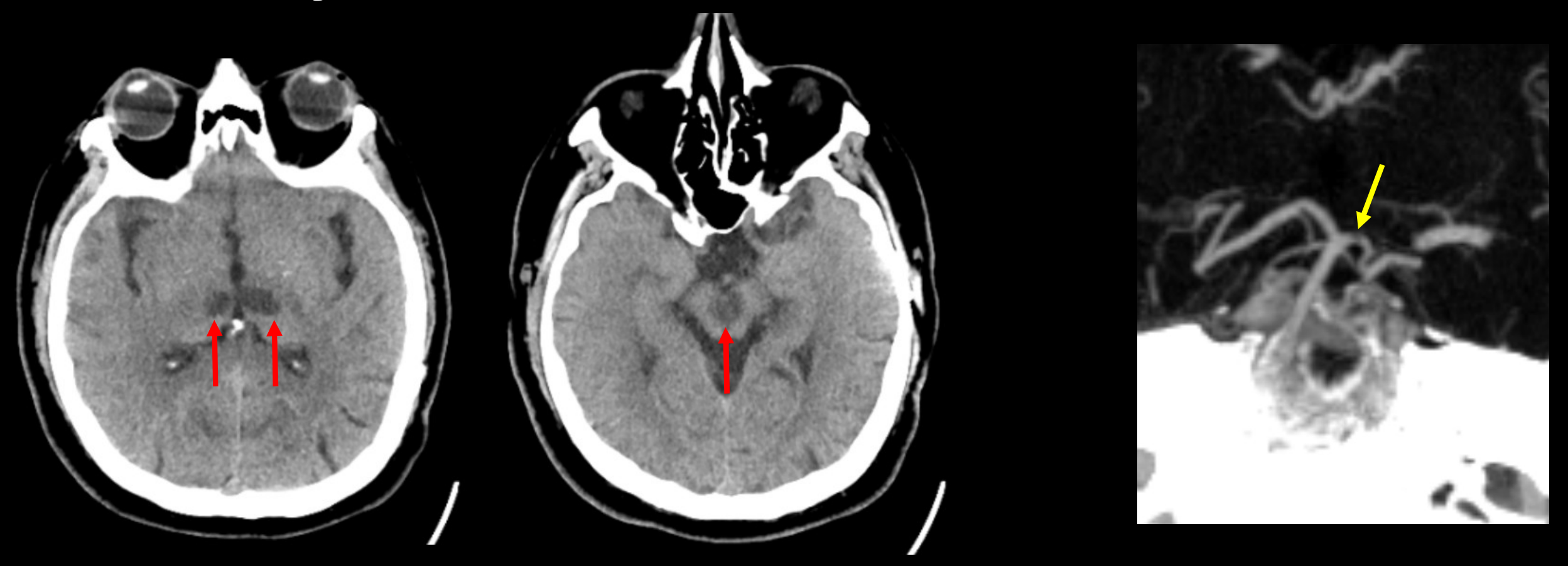

63 yo $M$, presenting after fall.

Infarcts in the bilateral medial thalami and superior midbrain.

Focal filling defect in proximal P1 segment of left posterior cerebral artery. 


\section{Choroid Plexus Infarct}

\section{- Anatomy}

- Blood supply to choroid plexus arises from anterior, posterolateral, and posteromedial choroidal arteries

- Rich collateral supply $\rightarrow$ rare to have choroid plexus infarcts

- Choroid plexus: produce CSF in brain

- However, isolated choroid plexus infarcts typically do not have any clinical significance or have a significant impact on CSF regulation

\section{- Differential diagnosis}

- Choroid plexus xanthogranuloma

- High signal on DWI, often bilateral

- Variable signal intensities depending on mixture of lipid, fluid, and blood products

- Intraventricular hemorrhage

- Dependent fluid-fluid levels in ventricles

- Incomplete CSF/FLAIR signal suppression

- Pyogenic ventriculitis

- Layering debris in occipital horns with marked restricted diffusion 


\section{Choroid Plexus Infarct}

69 yo $M$, presenting with new left-sided facial droop and right-sided weakness.

Acute infarct in the right choroid plexus. Additional infarcts of the body and splenium of the right corpus callosum.

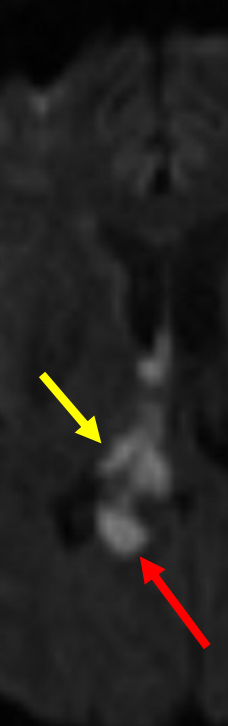

DWI

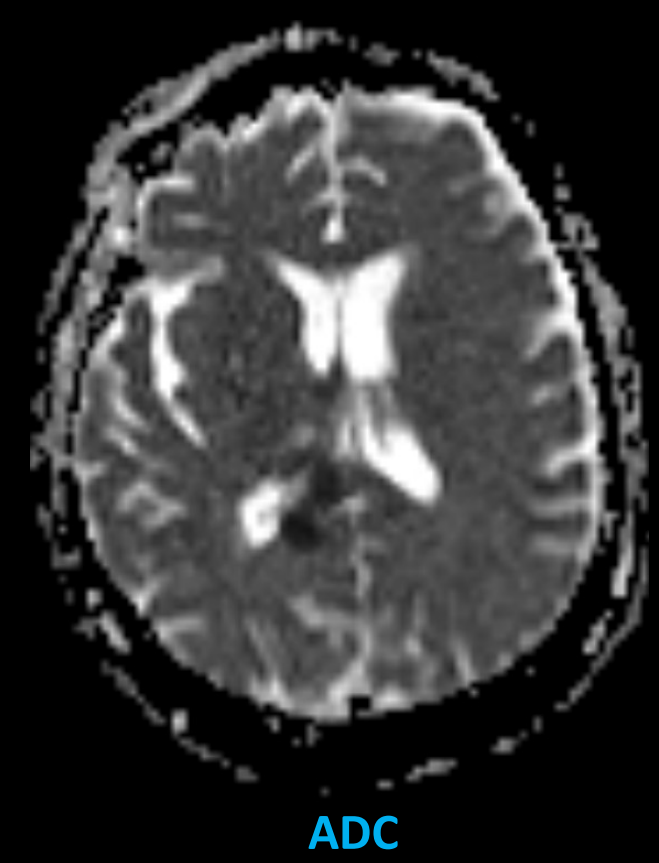




\section{Brainstem Stroke Syndromes}




\section{Internuclear Ophthalmoplegia}

- Anatomy

- Medial longitudinal fasciculus (MLF): paired white matter tracks in dorsomedial pontine and midbrain tegmentum near midline

- Convey signals from ipsilateral cranial nerve VI to contralateral cranial nerve III fibers $\rightarrow$ control medial rectus and allows for concerted eye movements

- Clinical Presentation: Defect in MLF $\rightarrow$ internuclear ophthalmoplegia

- Defect in MLF tract $\rightarrow$ ipsilateral medial rectus subnucleus of cranial nerve III not receiving signals from contralateral cranial nerve $\mathrm{VI} \rightarrow$ impaired ipsilateral adduction

- Weak ipsilateral medial rectus motion leads to excessive innervation of contralateral lateral rectus $\rightarrow$ contralateral horizontal nystagmus during abduction

- Etiology

- Brainstem demyelination (usually bilateral)

- Brainstem infarcts (usually unilateral)

- Others:

- Brainstem tumors, hemorrhage, trauma

- Progressive supranuclear palsy

- Infection
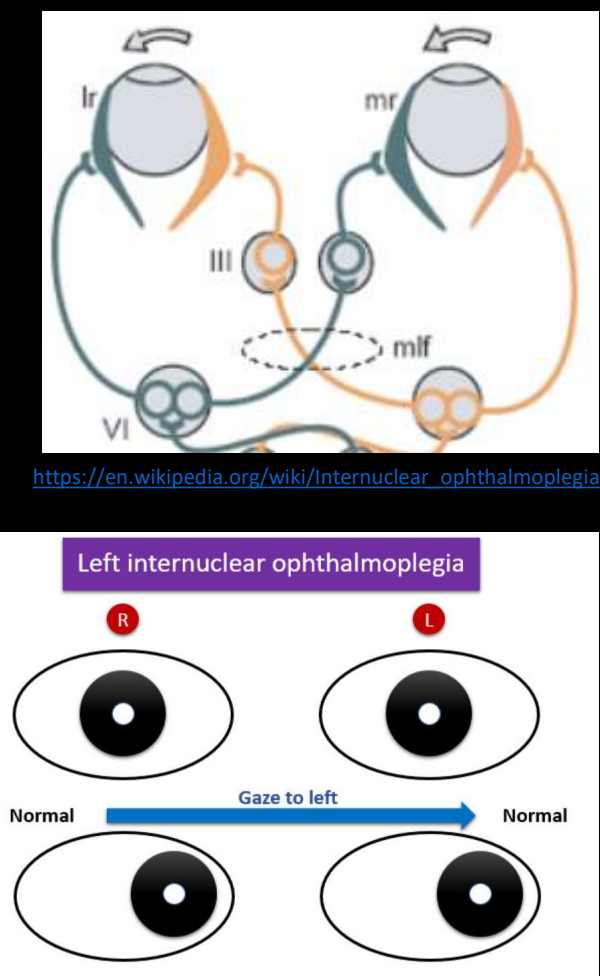

Gaze to right Loss of

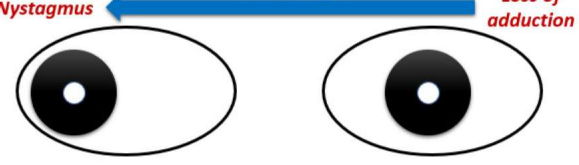




\section{Internuclear Ophthalmoplegia}

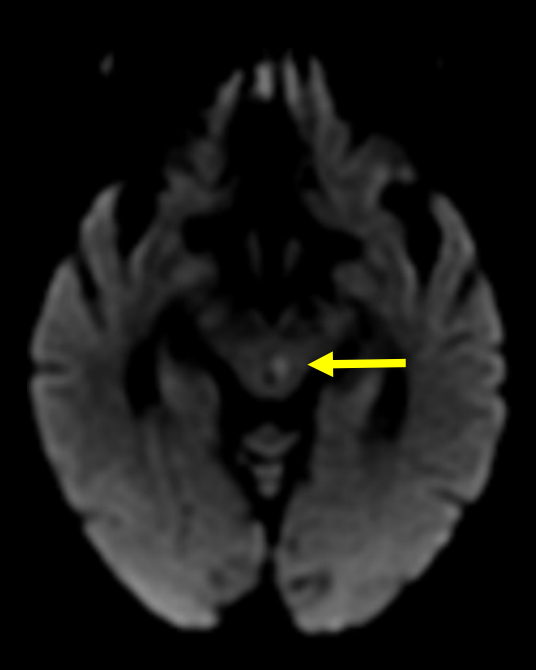

DWI

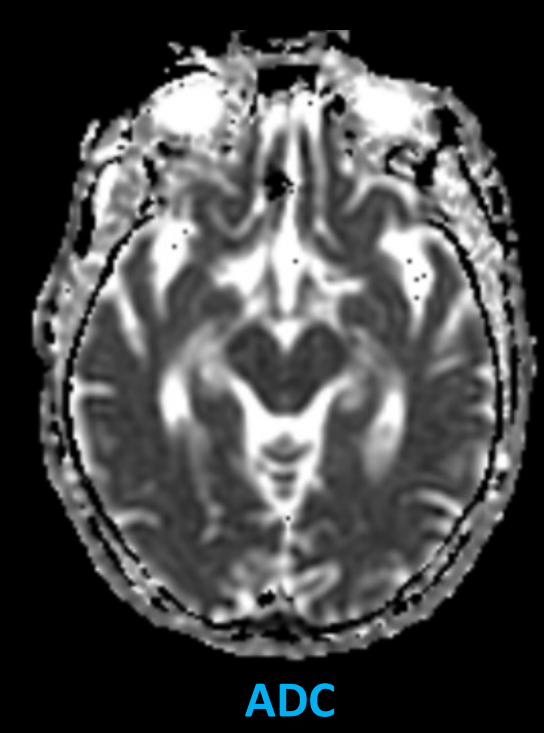

ADC

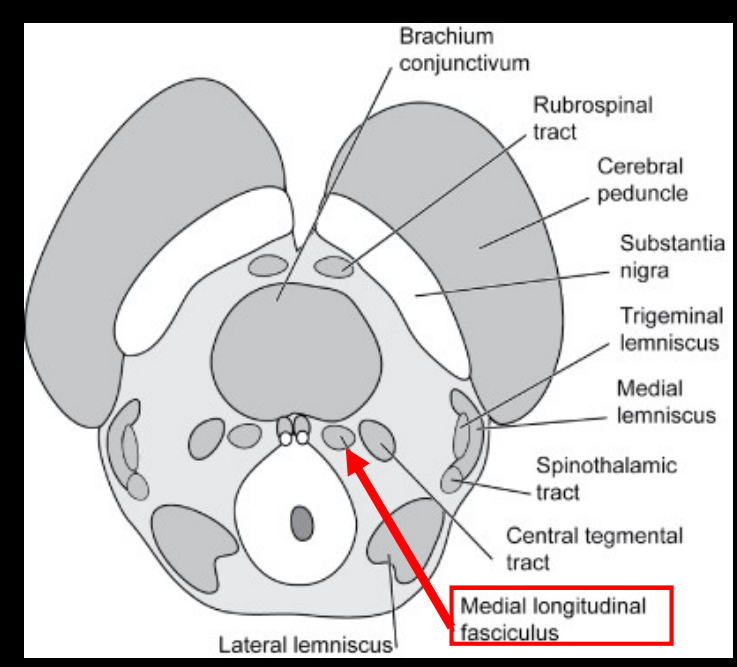

https://www.cambridge.org/core/books/clinical-neuroradiology/the-midbrain/55EA666FA62A40F0D220F575A562C5AB

90 yo F, presenting with double vision and left internuclear ophthalmoplegia.

Punctate acute infarct in the left midbrain anterolateral to cerebral aqueduct (region of the medial longitudinal fasciculus). 


\section{Facial Colliculus Syndrome}

- Anatomy

- Facial colliculus as anatomical elevation/bump in floor of $4^{\text {th }}$ ventricle

- Formed by fibers of facial nerve arching dorsally around abducens nerve

\section{- Clinical presentation}

- Peripheral facial nerve palsy (affects top and bottom of face) - Involvement of facial nerve fibers at genu of facial nerve

- Horizontal conjugate gaze palsy and diplopia

- Involvement of abducens nerve (unable to abduct ipsilateral eye)

- Involvement of paramedian pontine reticular formation located near abducens nucleus (loss of horizontal saccades towards side of lesion)

- Etiology

- Younger patients

- Tumor, demyelination, viral infection (rhomboencephalitis)

- Older patients

- Vascular infarcts
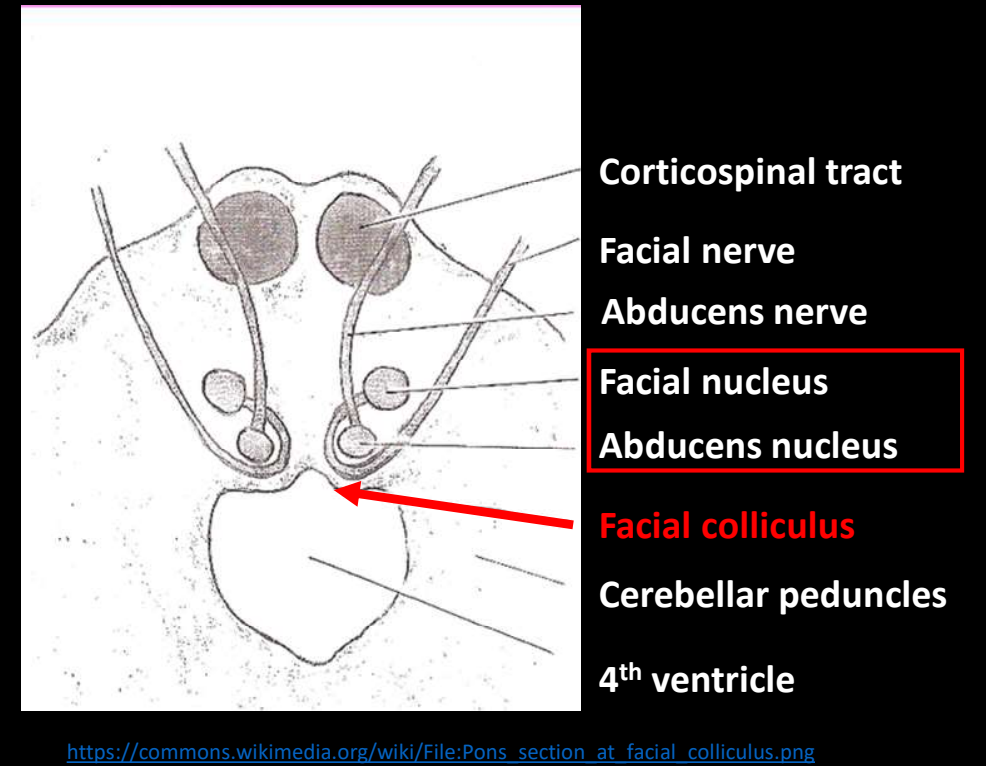


\section{Facial Colliculus Syndrome}
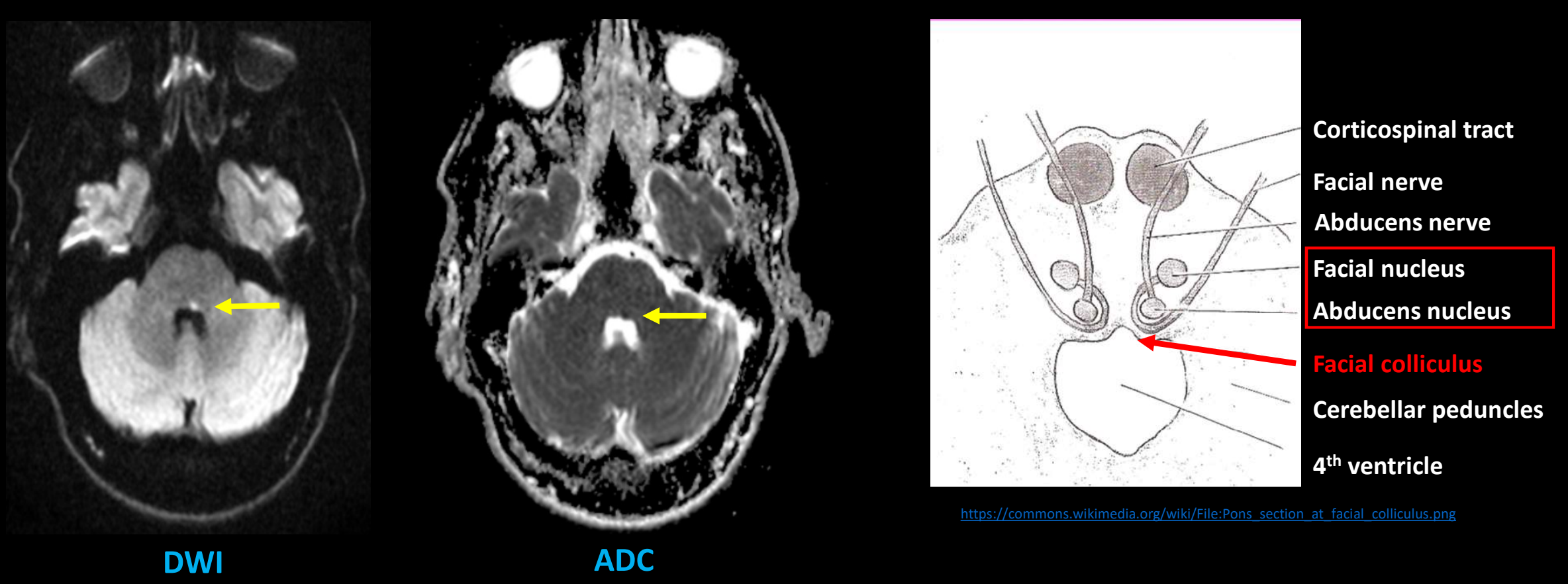

50 yo $M$, presenting with double vision, dizziness, and left $6^{\text {th }}$ nerve palsy.

Punctate acute infarct in the dorsal left paramedian pons (region of the left facial colliculus). 


\section{Lateral Medullary Syndrome (Wallenberg)}

- Anatomy of lateral medulla

- Olivary nuclei

- Spinothalamic tract

- Vagus nerve

- Clinical Presentation

- Ipsilateral cerebellar symptoms such as vertigo and diplopia (infarct of olivary nucleus)

- Crossed hemi-sensory disturbance involving ipsilateral face and contralateral body (infarct of spinothalamic tract)

- Ipsilateral Horner syndrome (infarct of vagus nerve)

- Etiology

- Occlusion of vertebral artery, PICA, or medullary arteries

- Cerebral embolism

- Vertebral artery dissection

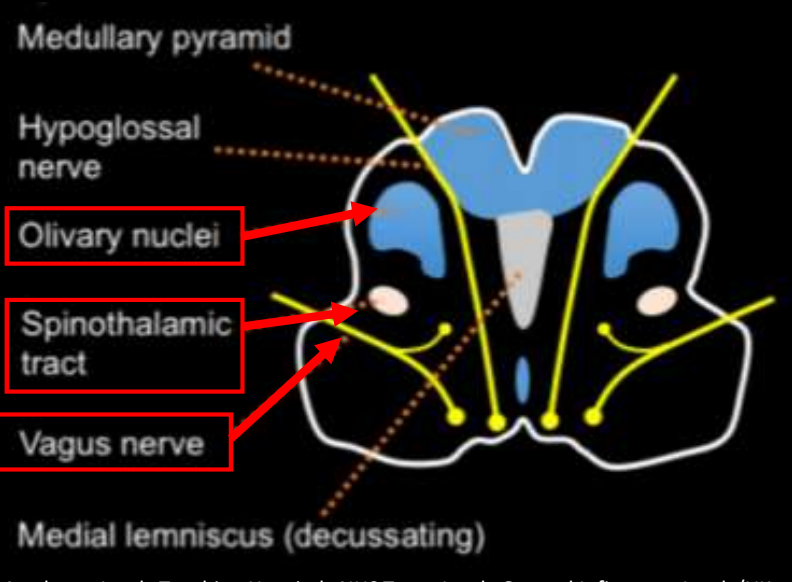

๑ Radiology Academy, Leeds Teaching Hospitals NHS Trust, Leeds General Infirmary - Leeds/UK 


\section{Lateral Medullary Syndrome (Wallenberg)}

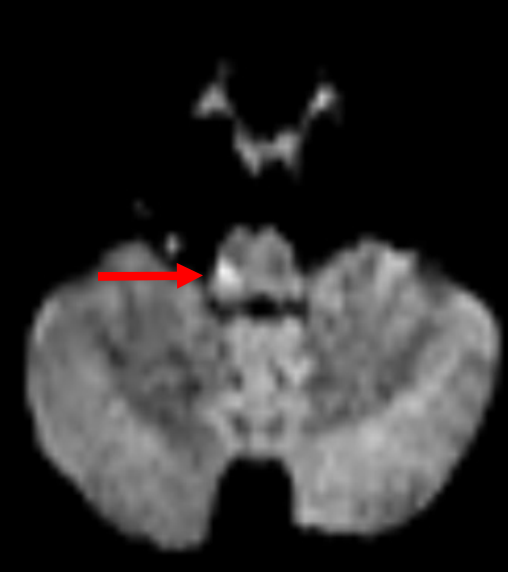

DWI

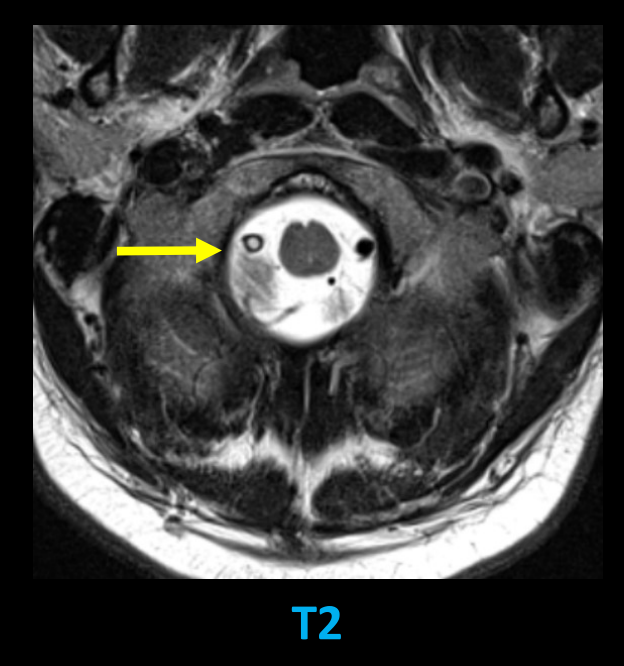

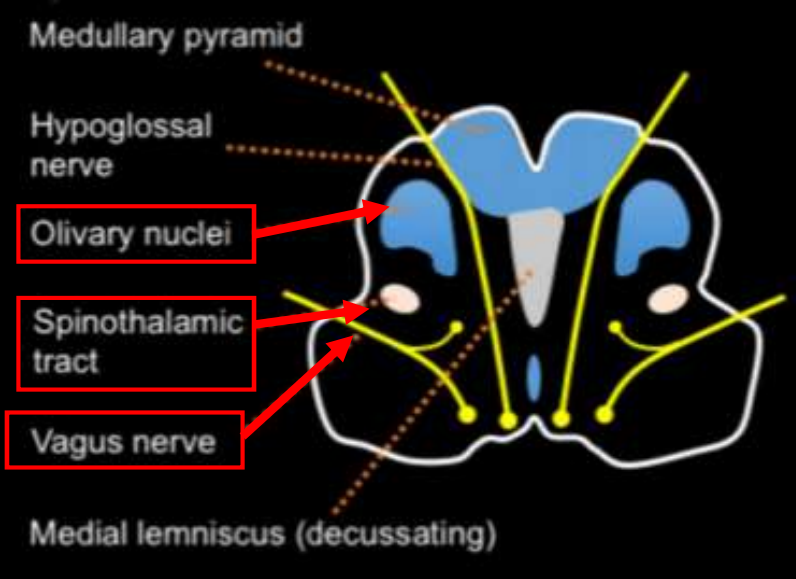

๑ Radiology Academy, Leeds Teaching Hospitals NHS Trust, Leeds General Infirmary - Leeds/UK

37 yo $\mathrm{M}$ with headaches, ataxia, dysphagia, and left arm and leg numbness after myofascial release treatment.

Acute infarct in the lateral right medulla centered in the spinothalamic tract. Abnormal flow void in the intradural segment of the right vertebral artery. 


\section{Lateral Medullary Syndrome (Wallenberg)}

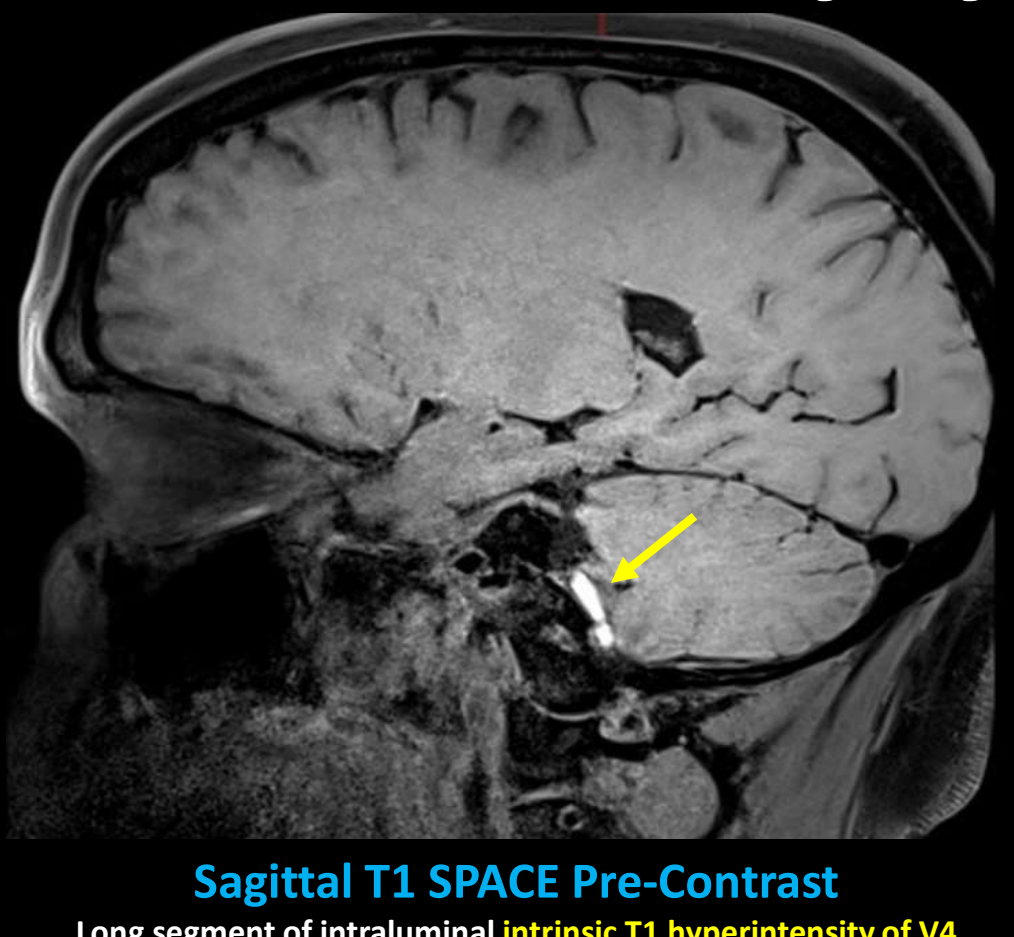

Long segment of intraluminal intrinsic T1 hyperintensity of V4 segment of right vertebral artery (intraluminal hematoma/thrombus)

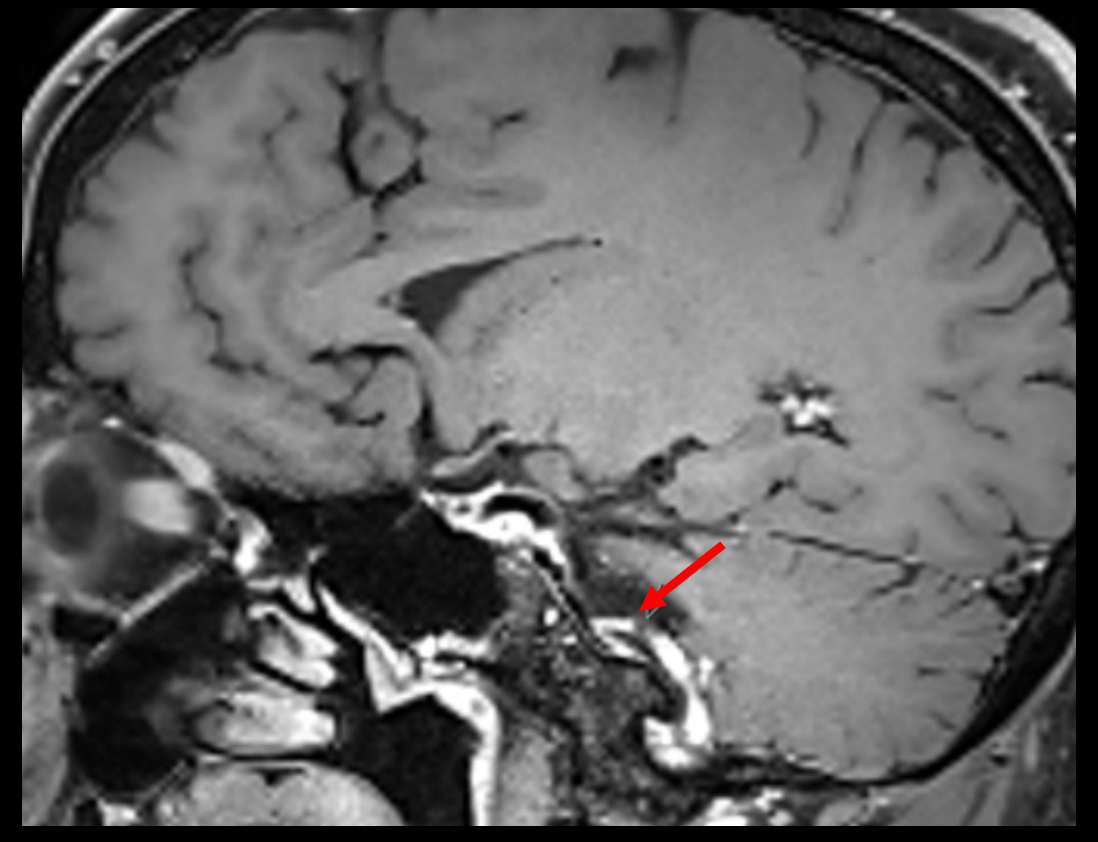

Sagittal T1 SPACE Post-Contrast

T1 hypointense line within V4 lumen, suggestive of intimal flap in the setting of right $\mathbf{V} 4$ segment vertebral dissection 


\section{Summary}

- Unusual brain infarct patterns can be challenging for both radiologists and clinicians.

- Familiarity with vascular anatomy, anatomic variants, patterns of uncommon infarctions, and clinical symptoms of brainstem stroke syndromes is critical for appropriate patient care. 


\section{Thank you!}

Stephanie.Wu@pennmedicine.upenn.edu 